\title{
Determination of a Common Clonal Origin of Gastric and Pulmonary Mucosa-Associated Lymphoid Tissue Lymphomas Presenting Five Years Apart
}

\author{
Norihiko Kawamata, Tohru Miki, Tetsuya Fukuda, Ken Suzuki, Yuki Sumi, Shinichi Ohdama, \\ Shinsaku Hirosawa, Osamu Matsubara*, Ryuichi KamiYama** and Nobuo AoKi
}

\begin{abstract}
Mucosa-associated lymphoid tissue (MALT) lymphoma is often mis-diagnosed as a benign tumor. Dissemination to other sites occurs in MALT lymphoma. We report a 60-year-old man with gastric and pulmonary tumors of MALT lymphoma which occurred 5 years apart. Initially, the gastric tumor had been diagnosed as reactive lymphoreticular hyperplasia. To determine whether the two tumors arose from the same malignant clone, we amplified and sequenced the complementarity-determining region 3 of the immunoglobulin heavy chain gene using the polymerase chain reaction (PCR). The sequences were identical except for 11-nucleotide difference, suggesting identical clonality.
\end{abstract}

(Internal Medicine 34: 220-223, 1995)

Key words: reactive lymphoid hyperplasia, polymerase chain reaction, complementarity-determining region 3

\section{Introduction}

Mucosa-associated lymphoid tissue (MALT) lymphoma is one of the low-grade B-cell lymphomas (1). It occurs in the gastro-intestinal tract, lung, salivary gland, and thyroid, and usually remains at the primary site (2). The disease is characterized by indolent progression leading to a prolonged clinical course (2). The tendency to remain at the primary site and specific dissemination to other mucosal sites are characteristic features of MALT lymphoma that are poorly understood. The diagnosis of MALT lymphoma is difficult to make sometimes, and MALT is frequently mis-diagnosed as reactive lymphoreticular hyperplasia (RLH) or some other benign tumor, as the abnormal centrocyte-like cells surrounding the normal reactive B-cell follicles are few.

Recent progress in molecular biological analysis has made it possible to detect small monoclonal B-cell populations in paraffin-embedded tissues using the polymerase chain reaction (PCR). In B-cell malignancies, amplification by PCR of the complementarity-determining region 3 (CDR3) that is generated by V-D-J joining of the immunoglobulin heavy chain gene is useful $(3,4)$ for the demonstration of monoclonality of the disease. By sequencing the CDR3 of separate tumors, it is possible to determine whether the malignant cells are derived from the same origin, since the pattern of V-D-J joining is very variable and is specific for each B-cell clone.

Here we report a patient who had MALT lymphoma in the stomach which was diagnosed initially as RLH and who developed the same type of MALT lymphoma 5 years later. We amplified and sequenced the respective CDR3 regions of the two specimens to confirm clonality.

\section{Case Report}

A 60-year-old man was admitted to an affiliated hospital complaining of epigastric discomfort. A large submucosal tumor was found in the stomach, and a gastrectomy was performed. The pathologic diagnosis of the excised tumor was RLH. The patient was followed without any further therapy.

Five years later he was readmitted to our hospital because of an abnormality seen on a chest roentgenogram ordered as part of a routine examination. Chest radiographs revealed alveolar shadows in the right lower lung field. As no pathogenic bacteria were detected on sputum culture, and treatment with antibiotics

From the First Department of Internal Medicine, *the Second Division of Pathology, and **School of Allied Health Sciences, Tokyo Medical and Dental University, Tokyo

Received for publication June 30, 1994; Accepted for publication November 25, 1994

Reprint requests should be addressed to Dr. Norihiko Kawamata, the First Department of Internal Medicine, Tokyo Medical and Dental University, 5-45, Yushima 1-chome, Bunkyo-ku, Tokyo 113 
did not show any improvement, a transbronchial lung biopsy (TBLB) was performed to evaluate the cause of the alveolar process. The pathologic diagnosis of the TBLB samples was MALT lymphoma. A lobectomy was performed.

\section{Materials and Methods}

\section{Pathologic analysis}

We reviewed formalin-fixed, paraffin-embedded sections of the gastric tumor and specimens of the excised lobe of the lung which were stained with hematoxylin and eosin (HE). The samples were obtained with informed consent.

\section{PCR analysis}

DNAs were extracted from paraffin-embedded samples of the gastric tumor using a method previously reported (5) and from the resected pulmonary lobe using the standard proteinase-phenol-chloroform method (6). Approximately $0.1 \mu \mathrm{g}$ of DNA was subjected to PCR (3). Semi-nested PCR was performed to amplify the CDR3 sequences, in which primers were directed to the joining region (first-round primer, LJH: 5'TGAGGAGACGGTGACC-3'; nested second-round primer, VLJH: 5'-GTGACCAGGGTNCCTTGGCCCCAG-3') and to the conserved framework-three segment of the variable region [5'-ACACGGC(C/T)(G/C)TGTATTACTGT-3'] (7, 8). The PCR consisted of 3 minutes pre-heating at $94^{\circ} \mathrm{C}, 40$ cycles of 1 minute denaturing at $94^{\circ} \mathrm{C}, 1$ minute annealing at $55^{\circ} \mathrm{C}$ and 1 minute extension at $74^{\circ} \mathrm{C}$, and a 10 minutes post-extension at $74^{\circ} \mathrm{C}$. The PCR was performed on a PC-700 thermocycler (Astec, Tokyo, Japan). Products were electrophoresed using a $12 \%$ polyacrylamide gel, and the gel was stained with ethidium bromide.

\section{Cloning and sequencing of $P C R$ products}

PCR products were ligated into pUC18 plasmid vectors using a Sure Clone PCR ligation kit (Pharmacia LKB, Tokyo, Japan). Nucleotide sequences were determined by fluorescence on an Autosequencer 373A (Applied BioSystem, Foster City, CA, USA) using vector-specific primers.

\section{Results}

The specimen of the gastric tumor which had been diagnosed initially as RLH showed centrocyte-like cells infiltrating around reactive B-cell follicles with destruction of the epithelial layers. These findings are compatible with the histologic features of a low-grade B-cell lymphoma of the MALT type (1) (Fig. 1A). Histologic examination of the resected lung also revealed MALT lymphoma with infiltration of medium-sized cells (Fig. 1B).

A single band representing rearrangement of the immunoglobulin heavy chain gene was detected in both PCR prod-

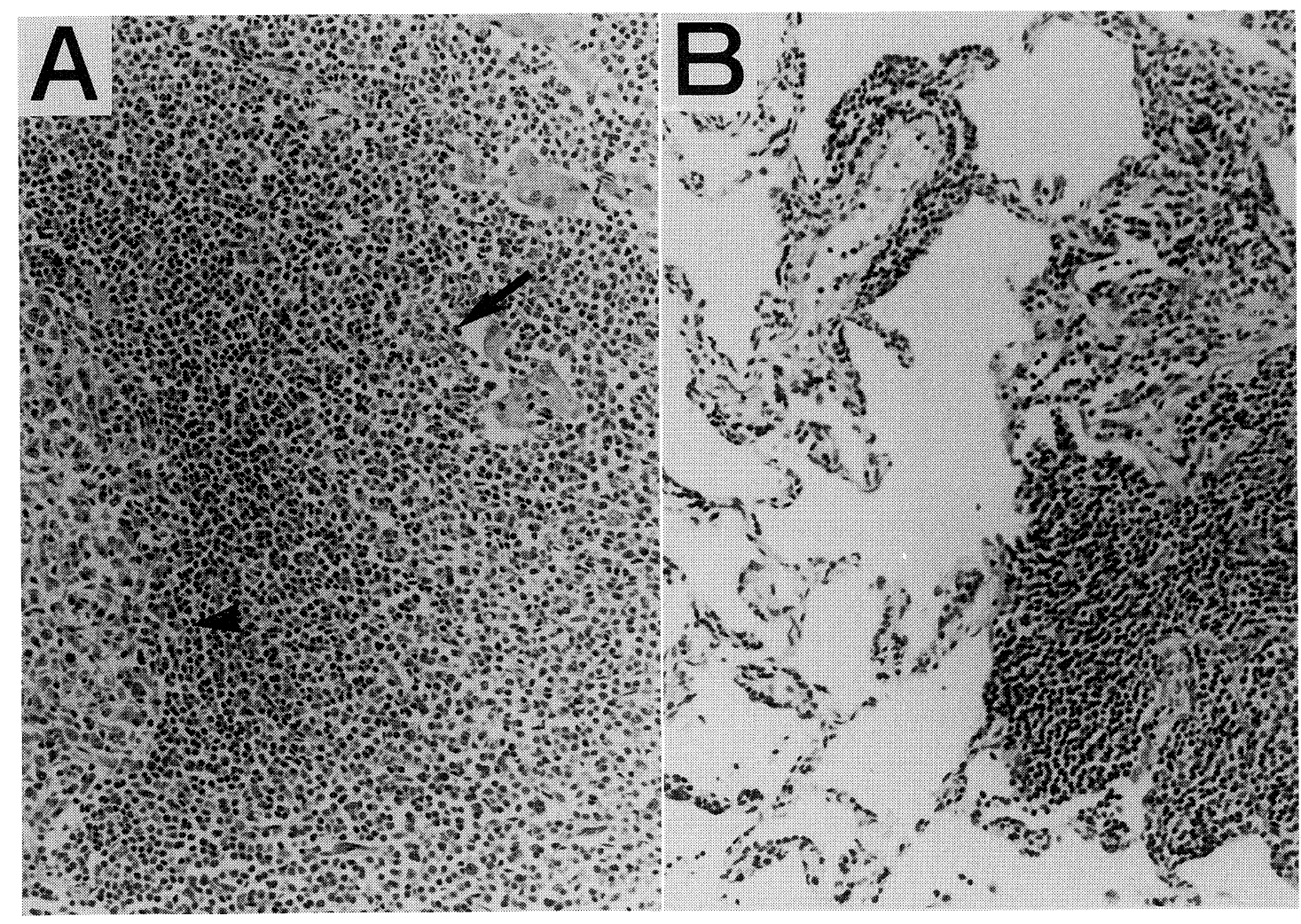

Fig. 1. The pathologic features of the two MALT specimens. Panel A is the stomach tumor. A reactive B-cell follicle (arrowhead) is surrounded by a diffuse infiltrate of centrocyte-like cells (arrow) (HE stain, $\times 100$ ). Panel B is the excised lung (HE stain, $\times 100$ ). Abnormal cells of medium size infiltrate the alveolar space. 


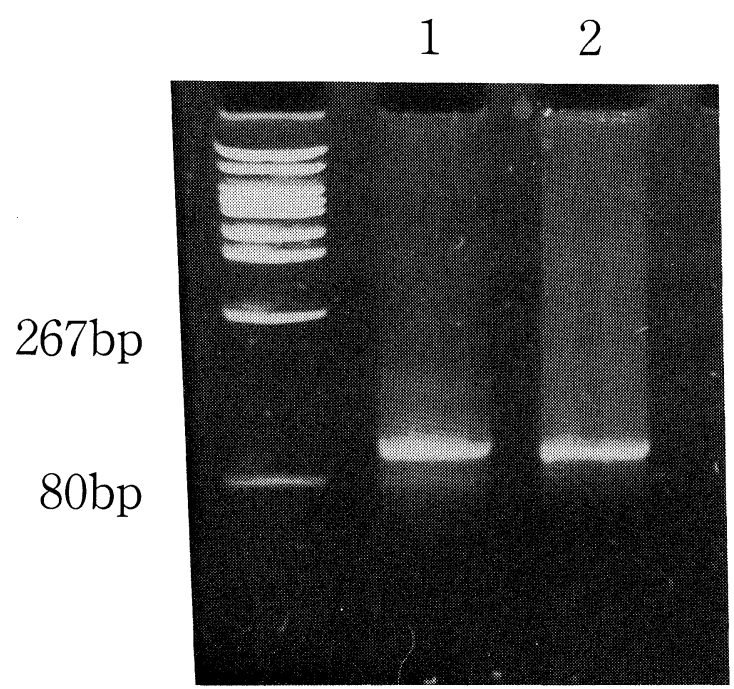

Fig. 2. Electrophoresis of PCR products on a $12 \%$ polyacrylamide gel. Twenty $\mu$ l of the PCR products were applied to each lane. Lane 1 is the PCR product of the stomach tumor and lane 2 is that of the pulmonary tumor. Single bands are detected in both lanes. The left lane is the PHY size markers (Takara, Kyoto, Japan). difficult to diagnose the disease correctly, and MALT lymphoma has been misdiagnosed frequently as other benign tumors (13). In reactive B-cell hyperplasia, infiltrating lymphocytes do not have monoclonality, so that PCR analysis shows a smear pattern $(9,14)$, while a single band is detected in MALT lymphoma. Therefore PCR analysis is a very useful method to distinguish MALT lymphoma from other benign tumors, including RLH.

The immunoglobulin variable region of the two tumors in this study showed differences between the stomach and lung samples in nucleotides at 11 positions. Since Taq DNA polymerase lacks a proof-reading function, some point mutations occur during the PCR procedure (5). The frequency of a substitution of a nucleotide during a single cycle extension of PCR is estimated at 1/8000 (5). As we carried out 40 cycles of extension, the mutation rate in our amplification was estimated at $1 / 200$. The number of point mutations found in our study was far more than that expected from the PCR procedure. Therefore, it is reasonable to assume that these mutations occurred during the development of the disease.

MALT lymphoma has been reported to be indolent, and to have a tendency to remain at the primary site. In the present

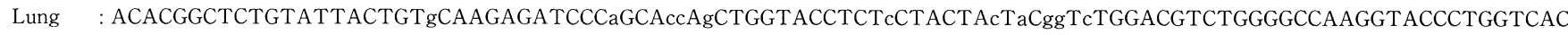

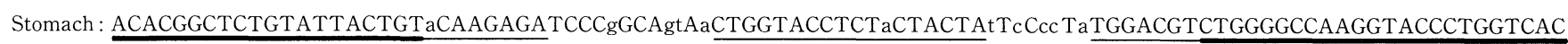
\begin{tabular}{llllll}
\hline FR3A & VH6 & $N$ & $\mathrm{~N}$ & $\mathrm{~N}$
\end{tabular}

Fig. 3. Nucleotide sequence of CDR3 of the samples. The upper sequence (Lung) is derived from the excised lung. The lower sequence (Stomach) is derived from the stomach tumor. The primer sequences (FR3A and VLJH) are shown with bold underlines. The variable region sequence (VH6), diversity region (D), and joining region (J6) are indicated by thin underlines. $\mathrm{N}$ segments $(\mathrm{N})$ have no underline. Nucleotide differences between the two sequences are indicated by lower-case letters.
\end{abstract}

ucts (Fig. 2). The similar intensity of both bands may be a result of the plateau effect in PCR amplification. These findings suggest that the malignant cells from both tissue are monoclonal $(8,9)$.

As the bands from both samples had similar migration patterns, the sequences were determined to see whether or not the origin of the two tumors was the same. The nucleotide sequences of the samples were found to be identical in the V-D$\mathrm{J}$ joining except for a difference of 11 nucleotides, one in the $\mathrm{V}$ region, one in the $\mathrm{D}$ region and nine in the $\mathrm{N}$ region (Fig. 3 ). The patterns of V-D-J joining (10-12) of these samples indicated that both tumors arose from a common clone.

\section{Discussion}

In this study we showed by PCR that a gastric MALT lymphoma which had initially been diagnosed as RLH, and a pulmonary MALT lymphoma which had developed 5 years later in the same patient arose from the same clone.

In MALT lymphoma, normal reactive B-cell follicles exist, and only a few abnormal centrocyte-like cells are seen surrounding the follicles. This pathohistologic feature makes it case, the patient developed a tumor in the lung, not in the primary site of the gastrointestinal tract. This indicates that dissemination to other sites occurs in MALT lymphoma. Blazquez et al (15) have reported that mucosal dissemination to other sites occurs more frequently than has been previously reported.

It is conceivable that there are many patients with MALT lymphoma who are mis-diagnosed initially with other benign tumors. In such cases, an initial correct diagnosis and appropriate treatment will improve the lymphoma patient's prognosis. When a patient is diagnosed as having reactive B-cell hyperplasia, it is important to rule out the presence of monoclonality in the tumor by PCR.

Acknowledgment: We thank Dr. Iwabuchi for providing clinical information and Miss Okada for technical support.

\section{References}

1) Isaacson PG, Wright DH. Malignant lymphoma of mucosa-associated lymphoid tissue. A distinctive type of B-cell lymphoma. Cancer 52: 1410, 1983.

2) Isaacson PG. Lymphomas of mucosa-associated lymphoid tissue (MALT). Histopathology 16: 617, 1990. 


\section{MALT Lymphoma}

3) Saiki RK, Scharf S, Faloona F, et al. Enzymatic amplification of betaglobin genomic sequences and restriction site analysis for diagnosis of sickle cell anemia. Science 230: 1350, 1985.

4) Yamada M, Hudson S, Tournay $O$, et al. Detection of minimal disease in hematopoietic malignancies of the B-cell lineage by using thirdcomplementarity-determining region (CDR-III)-specific probes. Proc Natl Acad Sci USA 86: 5123, 1989.

5) Wright DK, Manos MM. Sample preparation from paraffin-embedded tissues. in: PCR Protocols - A Guide to Methods and Applications, Innis MA, Gelfand DH, Sninsky JJ, White TJ, Eds. Academic Press, San Diego, 1990, p.153.

6) Maniatis T, Fritsch EF, Sambrook J. Molecular Cloning, a Laboratory Manual. Cold Spring Harbor Laboratory Press, Cold Spring Harbor, 1989, p. E.3.

7) Brisco MJ, Tan LW, Orsborn AM, Morley AA. Development of a highly sensitive assay, based on the polymerase chain reaction, for rare Blymphocyte clones in a polyclonal population. Br J Haematol 75: 163, 1990.

8) Trainor KJ, Brisco MJ, Story CJ, Morley AA. Monoclonality in Blymphoproliferative disorders detected at the DNA level. Blood 75: 2220, 1990.
9) Ono H, Kondo H, Saito D, et al. Rapid diagnosis of gastric malignant lymphoma from biopsy specimens: detection of immunoglobulin heavy chain rearrangement by polymerase chain reaction. Jpn J Cancer Res 84: $813,1993$.

10) Ichihara $Y$, Matsuoka $H$, Kurosawa $Y$. Organization of human immunoglobulin heavy chain diversity gene loci. EMBO J 7: 4141, 1988.

11) Buluwela L, Rabbitts TH. A VH gene is located within $95 \mathrm{~Kb}$ of the human immunoglobulin heavy chain constant region genes. Eur J Immunol 18: $1843,1988$.

12) Wasserman $R$, Yamada $M$, Ito $Y$, et al. VH gene rearrangement events can modify the immunoglobulin heavy chain during progression of B-lineage acute lymphoblastic leukemia. Blood 79: 223, 1992.

13) Spencer J, Diss TC, Isaacson PG. Primary B cell gastric lymphoma. A genotypic analysis. Am J Pathol 135: 557, 1989.

14) Diss TC, Peng H, Wotherspoon AC, Pan L, Speight PM, Isaacson PG. A single neoplastic clone in sequential biopsy specimens from a patient with primary gastric-mucosa-associated lymphoid-tissue lymphoma and Sjögren's syndrome. N Engl J Med 329: 172, 1993.

15) Blazquez M, Haioun C, Chaumette MT, et al. Low grade B cell mucosa associated lymphoid tissue lymphoma of the stomach: clinical and endoscopic features, treatment, and outcome. Gut 33: 1621, 1992. 